\title{
Supporting Information of IC060568U
}

\section{Enhanced NIR-Luminescence in an Erbium-Tetrafluoroterephthalate Framework}

\author{
Banglin Chen, ${ }^{\dagger} *$ Yu Yang, ${ }^{\ddagger}$ Fatima Zapata $^{\dagger}$, Guodong Qian, ${ }^{\ddagger} *$ Yongshi Luo, ${ }^{\S}$ \\ Jiahua Zhang, ${ }^{\S}$ Emil B. Lobkovsky ${ }^{\perp}$ \\ ${ }^{\dagger}$ Department of Chemistry, University of Texas-Pan American, Edinburg, TX 78541- \\ 2999, USA. \\ ${ }^{\ddagger}$ Department of Materials Science \& Engineering,State Key Laboratory of Silicon \\ Materials, Zhejiang University, Hangzhou 310027, P. R. China. \\ ${ }^{\S}$ Key Laboratory of Excited State Processes, Changchun Institute of Optics, Fine \\ Mechanics and Physics, China Academy of Science, Changchun 130021, P. R. China \\ ${ }^{\perp}$ Department of Chemistry and Chemical Biology, Baker Laboratory, Cornell University, \\ Ithaca, New York 14853-1301
}

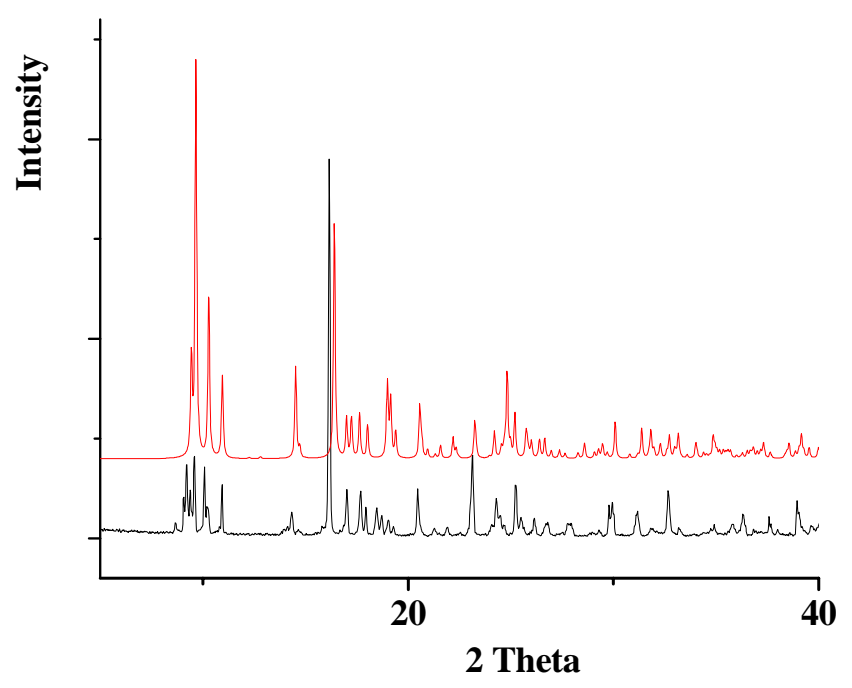

Figure S1. Powder X-ray diffraction (PXRD) patterns of as-synthesized compound $\mathbf{1}$ (black) and simulated patterns from X-ray single crystal structure of $\mathbf{1}$ (red). 
Table S1. Observed and Simulated PXRD of Compound 1

\begin{tabular}{|l|l|l|l|l|}
\hline \multicolumn{2}{|c|}{ Observed PXRD } & \multicolumn{2}{c|}{ Simulated PXRD } & \multicolumn{1}{c|}{ Indices } \\
\hline \multicolumn{1}{|c|}{ 2 Theta } & \multicolumn{1}{|c|}{$\mathbf{d}$} & \multicolumn{1}{c|}{ 2 Theta } & \multicolumn{1}{c|}{ hkl } \\
\hline 9.38 & 9.420 & 9.44 & 9.361 & 001 \\
\hline 9.59 & 9.215 & 9.66 & 9.148 & 010 \\
\hline 10.18 & 8.682 & 10.28 & 8.598 & 011 \\
\hline 10.94 & 8.081 & 10.96 & 8.066 & 100 \\
\hline 16.16 & 5.480 & 16.20 & 5.467 & $10-1$ \\
\hline 17.02 & 5.205 & 17.00 & 5.211 & $1-1-1$ \\
\hline 17.70 & 5.007 & 17.64 & 5.024 & 021 \\
\hline 20.56 & 4.316 & 20.56 & 4.317 & 122 \\
\hline 23.14 & 3.841 & 23.24 & 3.824 & 212 \\
\hline 25.22 & 3.528 & 25.2 & 3.531 & $2-11$ \\
\hline 29.94 & 2.982 & 30.08 & 2.968 & 133 \\
\hline 31.18 & 2.866 & 31.28 & 2.857 & 232 \\
\hline 31.82 & 2.810 & 31.82 & 2.810 & $2-21$ \\
\hline 32.66 & 2.740 & 32.72 & 2.735 & $1-22$ \\
\hline 33.18 & 2.698 & 33.16 & 2.699 & $1-13$ \\
\hline 34.92 & 2.567 & 34.86 & 2.572 & $3-10$ \\
\hline
\end{tabular}

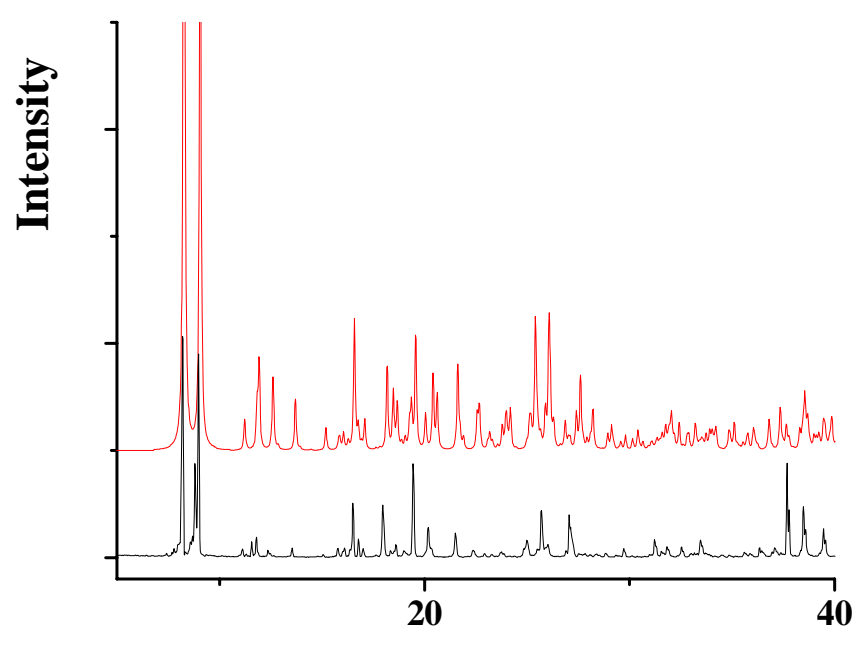

\section{Theta}

Figure S2. Powder X-ray diffraction (PXRD) patterns of as-synthesized compound 2 (black) and simulated patterns from X-ray single crystal structure of $\mathbf{2}$ (red). 
Table S2. Observed and Simulated PXRD of Compound 2

\begin{tabular}{|l|l|l|l|l|}
\hline \multicolumn{2}{|c|}{ Observed PXRD } & \multicolumn{2}{c|}{ Simulated PXRD } & \multicolumn{1}{c|}{ Indices } \\
\hline \multicolumn{1}{|c|}{ Theta } & \multicolumn{1}{c|}{ d } & \multicolumn{1}{c|}{ Theta } & \multicolumn{1}{c|}{ hkl } \\
\hline 8.20 & 10.77 & 8.26 & 10.70 & 010 \\
\hline 8.98 & 9.839 & 9.06 & 9.753 & $10-1$ \\
\hline 11.12 & 7.950 & 11.22 & 7.880 & $1-11$ \\
\hline 11.80 & 7.494 & 11.94 & 7.406 & $11-1$ \\
\hline 12.48 & 7.087 & 12.60 & 7.020 & $1-1-1$ \\
\hline 13.54 & 6.534 & 13.70 & 6.458 & 002 \\
\hline 15.06 & 5.878 & 15.18 & 5.832 & $02-1$ \\
\hline 15.76 & 5.618 & 15.86 & 5.583 & $1-12$ \\
\hline 16.50 & 5.369 & 16.58 & 5.342 & 020 \\
\hline 16.78 & 5.279 & 16.76 & 5.285 & $02-2$ \\
\hline 17.00 & 5.211 & 17.08 & 5.187 & 111 \\
\hline 17.96 & 4.935 & 18.18 & 4.876 & $20-2$ \\
\hline 18.34 & 4.833 & 18.48 & 4.797 & $12-1$ \\
\hline 18.60 & 4.766 & 18.66 & 4.751 & 012 \\
\hline 19.44 & 4.562 & 19.56 & 4.535 & $21-2$ \\
\hline 20.18 & 4.397 & 20.32 & 4.367 & $2-1-2$ \\
\hline 21.50 & 4.130 & 21.62 & 4.107 & $2-12$ \\
\hline 25.70 & 3.464 & 25.90 & 3.437 & $02-4$ \\
\hline 26.02 & 3.422 & 26.08 & 3.414 & $1-33$ \\
\hline 31.54 & 2.834 & 31.60 & 2.829 & $1-43$ \\
\hline 31.82 & 2.810 & 32.04 & 2.791 & 131 \\
\hline 33.46 & 2.676 & 33.52 & 2.671 & 040 \\
\hline 37.68 & 2.385 & 37.64 & 2.388 & $42-2$ \\
\hline 38.58 & 2.332 & 38.54 & 2.334 & $5-1-1$ \\
\hline 39.46 & 2.282 & 39.46 & 2.282 & $4-23$ \\
\hline & & & & \\
\hline
\end{tabular}




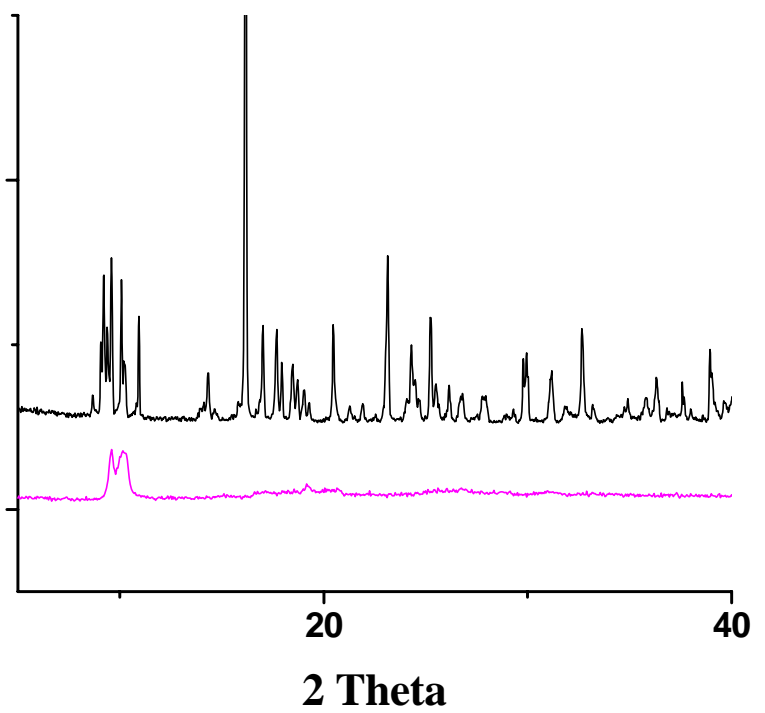

Figure S3. Powder X-ray diffraction (PXRD) patterns of as-synthesized compound $\mathbf{1}$ (black) and desolvated compound 1a (purple).

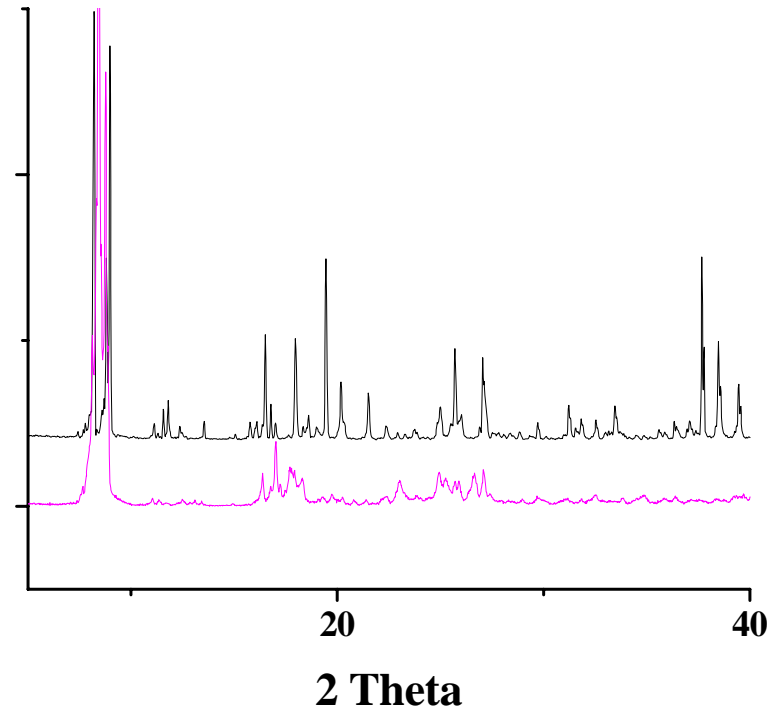

Figure S4. Powder X-ray diffraction (PXRD) patterns of as-synthesized compound 2 (black) and desolvated compound 2a (purple). 


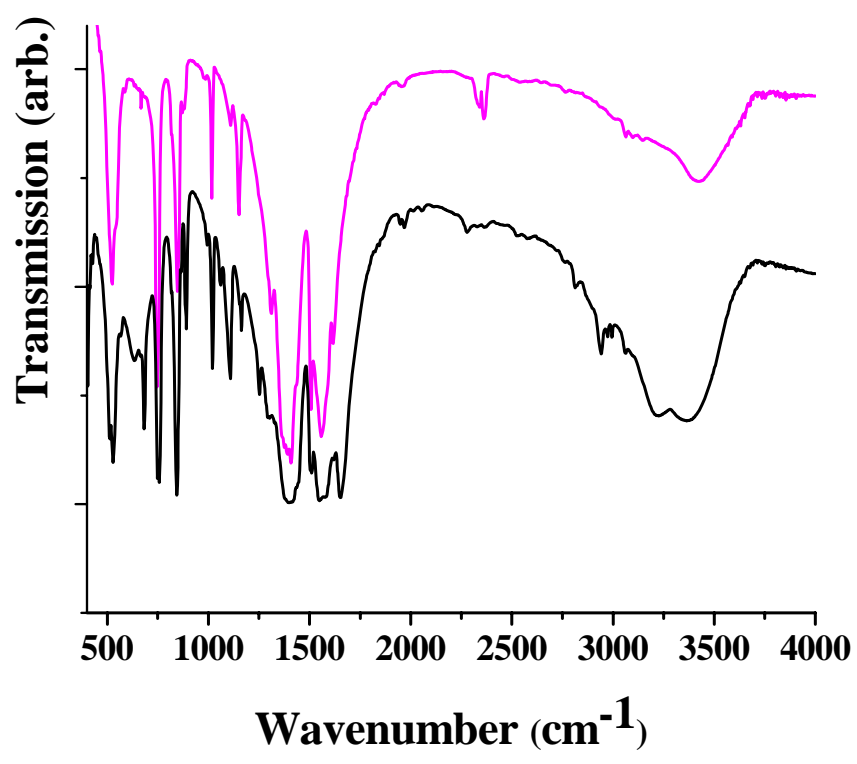

Figure S5. FTIR spectra of as-synthesized compound 1 (black) and desolvated compound 1a (purple).

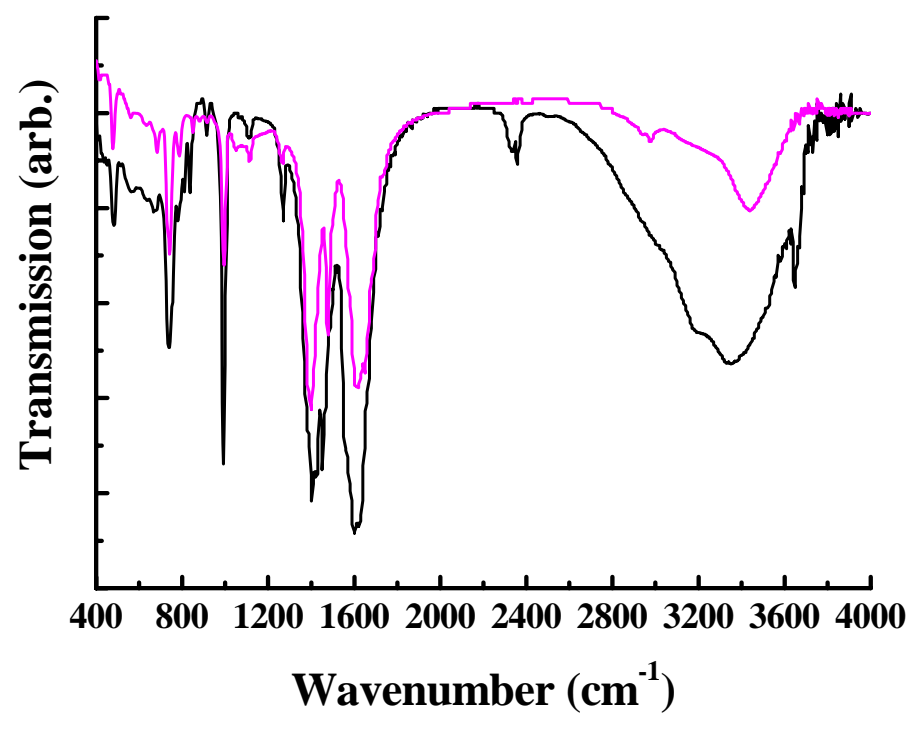

Figure S6. FTIR spectra of as-synthesized compound 2 (black) and desolvated compound 2a (purple). 